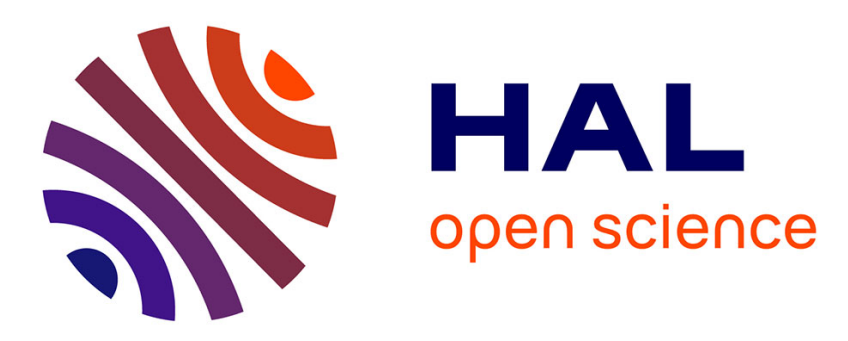

\title{
Physical principles and functional consequences of nuclear compartmentalization in budding yeast
} Judith Miné-Hattab, Angela Taddei

\section{To cite this version:}

Judith Miné-Hattab, Angela Taddei. Physical principles and functional consequences of nuclear compartmentalization in budding yeast. Current Opinion in Cell Biology, 2019, 58, pp.105-113. 10.1016/j.ceb.2019.02.005 . hal-02353853

\section{HAL Id: hal-02353853 https://hal.sorbonne-universite.fr/hal-02353853}

Submitted on 7 Nov 2019

HAL is a multi-disciplinary open access archive for the deposit and dissemination of scientific research documents, whether they are published or not. The documents may come from teaching and research institutions in France or abroad, or from public or private research centers.
L'archive ouverte pluridisciplinaire HAL, est destinée au dépôt et à la diffusion de documents scientifiques de niveau recherche, publiés ou non, émanant des établissements d'enseignement et de recherche français ou étrangers, des laboratoires publics ou privés. 


\title{
Physical principles and functional consequences of Nuclear compartmentalization in budding yeast.
}

\author{
Judith Miné-Hattab1,2 \& Angela Taddei1,2,* \\ 1 Institut Curie, PSL Research University, CNRS, UMR3664, F-75005 Paris, France. \\ 2 Sorbonne Université, Institut Curie, CNRS, UMR3664, F-75005 Paris, France. \\ * Corresponding author : Angela Taddei, Institut Curie - Centre de Recherche, 26, rue d'Ulm \\ 75248 Paris Cedex 05, France. Tel: +33156246704 angela.taddei@curie.fr
}

\begin{abstract}
: 115 words
One striking feature of eukaryotic nuclei is the existence of discrete regions in which specific factors concentrate while others are excluded, thus forming microenvironments with different molecular compositions and biological functions. These domains are often referred to as sub-compartments even though they are not membrane enclosed. Despite their functional importance the physical nature of these structures remains largely unknown. Here we describe how the Saccharomyces cerevisiae nucleus is compartmentalized and discuss possible physical models underlying the formation and maintenance of chromatin associated sub-compartments. Focusing on three particular examples: the nucleolus, silencing foci and repair foci, we discuss the biological implications of these different models as well as possible approaches to challenge them in living cells.
\end{abstract}

\section{Introduction to the yeast nucleus and its sub-compartments}

Like other eukaryotes, the genetic information of Saccharomyces cerevisiae yeast is separated from the cytoplasm by a double membrane forming the nucleus. This nucleus, whose radius is $\sim 1 \mu \mathrm{m}$ in $\mathrm{G} 1$ phase, contains the $\sim 13 \mathrm{Mb}$ of the haploid genome organized into 16 chromosomes [1], a genome nucleus ratio very similar to the average ratio in human cells.

\section{General organization of chromosomes:}

The 3-dimensional organization of the genome is well defined in budding yeast and decades of work in this organism has demonstrated the importance of this organization to regulate genome function [2]. In exponentially growing cells, Saccharomyces cerevisiae present a Rabl-like organization - in reference to the first description of the fold-back conformation of anaphase chromosomes by Carl Rabl in 1885 - in which all the centromeres remain attached to the spindle pole body through short microtubules during interphase, while telomeres are located close to the nuclear periphery [2-4] (Figure 1). Lying opposite to the centromeres is the nucleolus that is formed around the ribosomal DNA (rDNA) repeats located on chromosome XII. Although the global architecture of the genome is rather stable during interphase, local movements with amplitudes reaching $1 \mu \mathrm{m}$ within 10 seconds have been reported using live cell imaging [5]. Chromatin mobility is i) locus dependent, anchored loci such as telomeres, centromeres and the rDNA being less mobile than chromosomal middle arms [6, 7]; ii) strongly affected by growth conditions [8]; and iii) increased upon DNA damage $[9,10]$. 
Comparing chromatin mobility with different diffusion modes, previous studies reported confined diffusion $[5,6,11,12]$ while others have reported anomalous diffusion [1315]. However, these different studies have been performed at different time scales indicating that a single mode of diffusion is not sufficient to describe chromatin mobility [15]. Although variable, the apparent diffusion coefficient of chromatin is three orders of magnitude slower than the one of free fluorophores as estimated by FRAP or single molecule tracking (5.10-4 $\mu \mathrm{m} 2 / \mathrm{s}$ [12] to 1.10-3 [5] for chromatin versus $2 \mu \mathrm{m} 2 / \mathrm{s}$ for fluorescent proteins fused to NLS [16]. Several polymer models using a limited number of constraints, have been successfully used to recapitulate diverse experimental data on nuclear organization obtained by microscopy or chromatin conformation capture (HiC) [14, 17-20]. However, this organization is modulated by biological processes: some activated genes as well as unrepairable DNA damages associate with nuclear pores, and global reorganization of chromosome conformation are observed in different growth conditions [21-23] [24].

\section{Subnuclear Compartments:}

Chromatin occupies less than $3 \%$ of the nuclear volume, the rest being filled by millions of proteins [25]. One striking feature of the nucleus is the presence of domains called subcompartments, which are not membrane-enclosed and yet concentrate specific proteins while excluding others, thus forming microenvironments that favor or prevent particular activities. Particular examples are illustrated in Figure 1. Other sub-compartments or bodies such as PML, Cajal bodies speckles, and paraspeckles reported in metazoan cells are not detected in budding yeast. This could be owing to some specific features of the yeast genome (e.g. its small size, very compact structure, and lesser regulation potential) that make these subnuclear compartments dispensable. Furthermore, replication or transcription foci remain hardly detectable by conventional microscopy. These foci might be formed by a small number of proteins without creating a specific micro-environment. In the following section, we thus focus on 3 functional sub-compartments that are clearly visible by microscopy: the nucleolus, the silencing foci and repair foci.

\section{Focus on 3 nuclear sub-compartments: nucleolus, silencing foci and repair foci}

\section{The nucleolus:}

The nucleolus is the most prominent nuclear sub-compartment dedicated to ribobiogenesis. It is formed around the rDNA composed of 150-200 tandem copies of a $9.1 \mathrm{kbases}$ repeat ( 1.3 to $1.8 \mathrm{Mb}$ representing 10 to $14 \%$ of the yeast genome) located on chromosome XII and anchored to the nuclear envelope [26]. It presents a crescent-shaped structure that occupies around $30 \%$ of the nuclear volume in exponentially growing cells. The rDNA encodes ribosomal RNAs (rRNA) that are transcribed, processed, and assembled with ribosomal proteins (RP) to form pre-ribosomes within the nucleolus. If nutrients are not limited, cells can produce up to 2000 ribosome per minute. [27]. The nucleolus is thus particularly rich in proteins and is very dense as seen by electron microscopy. Substructures inside the nucleolus are visible on ultra-thin sections from cryofixed samples, possibly corresponding to zones of active transcription, RNA and pre-ribosome maturation [28, 29]. In addition to factors involved in ribo-biogenesis, the nucleolus is also storing factors involved in other cellular functions acting as a reservoir that can release these factors in a regulated manner. This has been well described for the Cdc14 phosphotase that is sequestered in the nucleolus until mitosis, when it is liberated to effect cell cycle progression [30]. More recently, nucleolar sequestration has been proposed to regulate the SUMO system and telomerase activity in budding yeast [31,32]. Conversely, some factors such as the recombination factors are excluded from the nucleolus [33]. 
Furthermore, some tRNA genes, which are scattered throughout the genome and transcribed by RNA PolIII, cluster at the boundary of the nucleolus in a transcription dependent manner [34, 35].

Assembly of the nucleolus is proposed to be a self-organized process, initiated by production of rRNA [36-38]. Accordingly, its size and morphology vary with ribosome production [39]. Moreover, the nucleolus undergoes major changes in shape and size in certain genetic contexts or physiological conditions. It fragments upon replicative aging when extra chromosomic rDNA circles accumulate as byproducts of homologous recombination events [40][41, 42]. In addition, in diploid yeast containing 2 rDNA loci, or when the rDNA is split into 2 chromosomes in haploid yeast, a single nucleolus is formed probably by the fusion of 2 initial nucleoli formed around each rDNA locus. However, when rDNA copies are split between multiple plasmid copies, micro nuclei are observed reminiscent to the situation observed in old mother cells [42]. The nucleolus is thus a very plastic sub-compartments adapting its shape to the cell's needs, with the ability to split and fuse, to sequester specific factors while excluding others.

\section{Silencing foci:}

Unlike other eukaryotes containing centromeric heterochromatin, budding yeast chromosomes have very little repetitive sequences outside of the rDNA. Transcriptionally inert and compacted chromatin is mainly found close to the TG-repeat DNA of telomeres. During exponential phase, the 32 telomeres of haploid cells accumulate at the nuclear envelope forming 3-5 foci, whose sizes are below the diffraction limit of conventional epifluorescence microscopes. These foci concentrate the silent regulatory factors Sir2, Sir3 and Sir4 that form the SIR complex [43]. In vitro, this complex associates with nucleosome with 1:2:1 stoechiometry and compacts chromatin [44]. Once recruited at the telomeres through interaction with the telomeric protein Rap1, this complex has the ability to spread on chromatin, and repress underlying genes [45], possibly by forming a compact chromatin structure [44].

Silent chromatin sequestration of SIR proteins in perinuclear foci favors subtelomeric repression and prevents the promiscuous binding of the SIRs at a distinct subset of promoters elsewhere in the genome [46-48]. Furthermore, the physical proximity of telomeres within these foci favors faithful recombination events upon double strand break [49].

The telomere composition of these foci is not fixed but telomeres belonging to chromosome arms of similar length show preferential association [50-52]. These compartments were also proposed to result from a self-assembly process owing to the properties of the SIR complex [53]. Reversible telomere anchoring to the nuclear periphery is achieved by Sir4 and other telomeric proteins that bind nuclear envelope associated proteins [53]. The grouping of telomeres in a limited number of foci is independent of heterochromatin formation but requires Sir3 association to telomeres [54]. Furthermore, these foci fuse into bigger foci or hyperclusters when SIR3 is overexpressed, indicating that Sir3 is a limiting factor for telomere clustering [54]. The bifunctional properties of Sir3 and Sir4 in silencing and respectively in clustering and perinuclear anchoring thus provide a mechanism for the self-establishment and propagation of a silent compartment [53].

Interestingly, silencing foci are dynamic: they fuse and divide in interphase and dissociate partially in metaphase to reform in G1 [51, 55]. More surprisingly, following carbon source exhaustion, silencing foci of long-lived quiescent cells relocalize to a unique "hypercluster" located in the nuclear center, a reorganization that contributes to the long-term viability of these cells [21]. Although this hyperclustering is very similar to the one observed upon SIR3 overexpression [54], here it is not accompanied with increase in Sir3 levels and the mechanisms driving such a major reorganization is not understood so far. 


\section{Repair foci:}

Repair foci are formed following double strand breaks (DSB) when repair proteins relocalize from a diffuse nuclear distribution to a highly concentrated sub-compartement at the damaged site [56]. Eukaryotic organisms use two major mechanisms to repair DSBs: nonhomologous end-joining (NHEJ) and homologous recombination (HR). Here, we will focus on repair sub-compartments formed by HR proteins. In eukaryotes, HR is orchestrated by multiprotein complexes forming mega-Dalton protein complexes of 500 to 2000 proteins at the DSB [56]. These micro-environments exhibit a concentration of proteins 50 times greater than in the rest of the nucleus. Upon DSB, the 5' ends of the DNA break are resected to yield 3'end singlestranded DNA (ssDNA) tails on which several recombination proteins including, RPA, Rad52 and Rad51, are recruited. Rad51 polymerizes on the damaged ends to form a nucleofilament that has the capacity to localize and invade a homologous DNA sequence $[57,58]$. In vitro, Rad51 proteins form a right-handed helix around DNA [59] inside which DNA is stretched by a factor of 1.5 compared to the B-DNA form [60]. This complex is extremely stiff, exhibiting a persistence length ranging from 300 to $600 \mathrm{~nm}$ [61] (compared to $60 \mathrm{~nm}$ for naked dsDNA). Given that resection was estimated to proceed at $4-5 \mathrm{~kb}$ per hour on each side of a DSB [62], one expects to observe rods in the range of $\mu \mathrm{m}$ rapidly after inducing an irrepairable DSB. In contrast, in vivo, recombination proteins form foci, whose size is under the diffraction limit of conventional microscopes.

Repair foci formed on damaged DNA are highly mobile. Indeed, damaged chromatin, and consequently the repair focus formed there, explore a nuclear space up to 10 times larger than before damage $[5,63]$. Such a dramatic change in mobility depends on the DNA damage checkpoint proteins $\operatorname{Rad} 9$ and Mec1, the repair protein Rad51 and the chromatin remodeler Ino80 $[9,10]$. However, chromatin diffusion coefficient is not significantly altered after DSB. Instead, changes in chromatin mobility enlarge the space that damaged chromatin explores without increasing the speed at which it moves [5].

Several studies indicate that multiple DSBs cluster together into a single "repair center". Although clustering unrelated DSBs might promote translocations, repair centers might create a microenvironment that favor repair efficiency or regulate the accessibility of nuclear factors to DSB ends [64-67].

\section{The physical nature of subnuclear-compartments}

As discussed above, subnuclear compartments are dynamic structures that have the potential to regulate many different processes by sequestering, or excluding specific activities around specific part of the genome. How these membrane-less sub-nuclear compartments are formed and maintain and what determines their size, shape, and number under different physiological conditions remain unclear. Several models have been recently proposed for the formation of membrane-less compartments [68-70]. Here we will consider 3 simplified models for the formation of chromatin associated compartments, the "binding model", the "bridging model" and the "droplet model", the 2 later also being referred to as "polymer-polymer phase separation model" and "liquid-liquid phase separation model" respectively [69]. We will then discuss their biological consequences as well as possible predictions for how to test their validity in living cells.

\section{Binding, bridging and droplet models}

A common feature of chromatin-associated sub-compartment is that they form around loci displaying multiple binding sites for specific factors. These multiple binding sites can be repeated sequences, arrays of nucleosomes bearing a specific histone mark or stretch of ssDNA 
(in the case of repair foci). A very simple model, could thus be the "binding model", in which sub-compartments are formed by the ensemble of proteins that bind available chromatin sites with a certain affinity and by surrounding proteins that are detached from their binding sites (Figure 2A). Such sub-compartments do not exhibit any phase separation. Their existence relies only on the protein's binding properties and on the number of binding sites on chromatin. Yet this type of subnuclear compartments can sequester specific factors because of the high avidity resulting from the density of binding sites.

If in addition, proteins are able to bridge distant chromatin loci by creating loops or by stabilizing interactions between distant loci along the chromatin fiber or belonging to different chromosomes, a "polymer-phase separation" can emerge (Figure 2B). These interactions can be driven by specific or multivalent weak interactions between chromatin binding proteins or chromatin components [71]. In this case, the existence of sub-compartments relies on both the binding and bridging properties of these proteins. Furthermore, polymers in bad solvents also phase separate [72] and chromatin phase separation could arise from a change in solubility of the surrounding molecular environment. Hult et al. proposed that the rDNA phase separates due to crosslinking, inducing the formation of a gel stiffer than the rest of the genome [73]. Similarly, interactions between Sir3 molecules have been proposed to bridge telomeres to form silencing foci $[53,54]$.

An alternative attractive hypothesis is that membrane-less compartments arise from a liquid-liquid phase separation and form droplets [74] (Figure 2C). Unlike the binding/bridging model, in the droplet model, proteins self-organize into liquid-like droplets that grow around the chromatin fiber allowing certain molecules to become concentrated while excluding others. Importantly, there is often a confusion between polymer-phase separation and liquid-phase separation: one should keep in mind that a phase separation can emerge from polymers without necessarily being in a liquid-phase $[72,75]$. Liquid-phase separations have been proposed to explain the formation of several nuclear sub-compartments such as heterochromatin in human and Drosophila cells [76, 77], and repair [78, 79] or transcription foci [80]. Since subcompartments are composed of many different proteins with distinct properties, one can also imagine that certain chromatin-bound proteins form a polymer-phase separation [73], while other proteins in the same sub-compartment form a liquid droplet. This is possibly the case for the nucleolus which has not only been described as a polymer-phase separation [73] but also as a liquid [81].

Although these different types of sub-compartments can be difficult to distinguish by current conventional microscopy techniques, their physical nature has important functional consequences.

\section{Functional consequences of the different models}

The essential hallmark of liquid-phase separation is its ability to exclude specific factors depending on their biochemical properties. Importantly, some large molecules are able to penetrate sub-compartments to perform their activity, while smaller proteins can be excluded. Liquid-like sub-compartments can thus generate a specific biochemical environment favoring or impairing specific reactions. Furthermore, access or exclusion of specific factors can be regulated by post-translational modifications increasing the regulatory potential of these types of subnuclear compartments [82]. In contrast, for both the binding and bridging models, access inside a domain is only limited by the steric hindrance; therefore, proteins are excluded from a sub-compartment depending on their size. Interestingly, recombination factors were shown to be excluded from the nucleolus and heterochromatic sub-compartments respectively in yeast [33] and Drosophila [65]. Such exclusion was proposed to prevent rearrangements between repetitive DNA sequences present in heterochromatin. As different types of sub-compartments 
have different regulatory potentials, it is important to define criteria allowing them to be distinguished.

\section{Distinctive Criteria for the different models}

Pioneers studies define membrane-less sub-domains as liquid droplets according to 3 criteria: i) their spherical shape, ii) their ability to rearrange and iii) their ability to fuse $[77,79$, 83]. However, polymer-phase separation also has the ability to fuse [71, 72]. The spherical criterium, originally predicted for liquid-phase separation $[79,83]$, is not validated in several studies describing heterochromatin sub-compartments as liquid-phase droplets [77], possibly due to shape constraints imposed by the elasticity of the heterochromatic polymer. Furthermore, as discussed above for repair or silencing foci, the shape of subnuclear compartments is often not accessible by microscopy. There is thus a need to refine the criteria allowing the different models mentioned above to be distinguished [69]. The emergence of single molecule microscopy opens the possibility to refine the predictions of each model at the microscopic level and to validate them experimentally.

\section{$>$ Internal structure of sub-compartments:}

A feature of the binding/bridging models is the presence of proteins mainly at chromatin binding sites. Thus, inside sub-compartments, we would expect to observe a non-homogenous distribution of proteins following the chromatin fiber. In contrast, in the droplet model, a microenvironment emerges inside which proteins fill the space of a sub-compartment homogenously, creating a sharp boundary between the interior and the exterior of a subcompartment. Super-resolution approaches will be instrumental to access the internal structure of small sub-compartments such as repair or silencing foci. However, deciphering the internal structure of sub-compartments might not be sufficient to discriminate bridging from droplet models. Indeed, liquid-phase separations are often discussed as not pure liquid droplets containing some bindings interactions serving as the nucleation core [74].

\section{Diffusion properties of proteins in the different models}

Another approach consists in studying the diffusion properties of proteins using, for example, fluctuation correlation spectroscopy or Single Particle Tracking (SPT). In the binding/bridging models, proteins inside sub-compartments should diffuse with an apparent diffusion coefficient very close to the one measured for chromatin. In contrast, the droplet model predicts that proteins can diffuse faster than chromatin, since even in the presence of a nucleation core, the large majority of proteins would not be bound to chromatin (Figure 2C). Interestingly, SPT measurements reveals that the silencing protein Sir3 and the repair proteins Rad52 have very different behaviors when observed at the single molecule level. Sir3 stays mainly bound to chromatin, while Rad52 diffuses faster inside a focus, suggesting that silencing foci would follow the bridging model and repair foci, the droplet model (Miné-Hattab et al., Sridhar et al., manuscripts in preparation). The confinement properties of trajectories are also expected to differs in the different models. In the droplet model, individual molecules should be confined within a volume reflecting the size of the droplet while in the binding/bridging models, bound molecules should follow the confinement of chromatin. Finally, the profile of diffusion coefficient inside a sub-compartment also differs between each model. In the binding/bridging model, the profile of diffusion coefficients through a sub-compartment should more or less follow the shape of the chromatin contained inside. In contrast, we expect to see a more homogenous and higher apparent diffusion coefficient inside a liquid droplet, with a sharp difference when a protein escapes the droplet. However, in the case of small sub-compartments, the latest prediction might be at the limit of the possible experimental resolution, in particular in yeast nuclei. 


\section{Impact of interfering with specific and non-specific interactions}

Despite their small size, yeast silencing and repair foci are yet good model systems to study the physical nature of sub-nuclear compartments owing to $i$, their reduced complexity compared to their functional equivalent in metazoans; $i i$, the knowledge accumulated over decades of genetic studies coupled with biochemistry and in vivo microscopy and iii, the ease to interfere with this biological system in rapid and well-controlled manner.

The different models of sub-compartments rely on different biochemical properties of their components: while the droplet model relies on non-specific, weak multivalent interactions often associated with disordered protein domains, the bridging model relies on more specific proteinprotein and/or DNA protein interactions. Deleting intrinsically disordered protein domains or using mutants known to interfere with specific protein-protein or protein DNA-interactions will be instrumental to identify the biochemical interactions underlying these compartments. Finally, one difficulty to study sub-compartments is their complex composition and the multiple interactions between the molecular components. Indeed, the permanent absence of individual components can prevent the formation or the maintenance of the entire structure. Inducible degradation is thus necessary to study the role of individual factors in either the formation or maintenance steps.

\section{Impact of changing the concentration of constituting protein factors:}

As discussed above the sphericity is not always a discriminant criterium. However, the binding/bridging models implicate that the size of the sub-compartments depends on the number of binding sites while in the droplet model, a large reservoir of proteins form independently of the number of binding sites. Mutants affecting the number of binding sites are available for repair (e.g. mutant affecting resection) and silencing (e.g. mutant affecting telomere length) foci in yeast that should help discriminating between the different models. Reciprocally, changes in protein amounts have different consequences in each model as discussed in [69]. Indeed, in the bridging model, increasing the concentration of proteins should first reduce the size of sub-compartments since more bridges are formed provoking chromatin to collapse more. However, after reaching a saturation point, the size of sub-compartments will plateau and only the nucleoplasmic concentration outside of the sub-compartments will increase. Conversely, in the droplet model, increasing protein amounts should increase the size of the droplet and the concentration will remain constant both in the nucleoplasm and in the droplet. According to this criterium, quantification of the Sir3 distribution when expressed at various levels is in agreement with the bridging model for telomere clustering [84].

\section{Conclusion}

The existence of membrane-less sub-compartments allows essential biological processes to be achieved at specific places and within precise time windows in the nucleus. Understanding how such microenvironments are created, maintained and disassembled is a very exciting challenge both for cell biologists and for bio-physicists. Although we mainly discussed these questions in the context of budding yeast nuclear organization that is the focus of this review, the general physical principles must apply to chromatin-based sub-compartments in all eukaryotes.

Faint foci such as replication or transcription sites in budding yeast could correspond to the binding model with a small number of proteins associated with the chromatin. Experimental data obtained in yeast indicate that silencing foci would result from the bridging model, while repair foci exhibit many features of liquid droplets. However, these models are not exclusive of one another. Binding events probably form the nucleation core of sub-compartments around which some proteins can form a liquid droplet. Furthermore stabilizing interaction within a 
droplet can convert the sub-compartment from a liquid to a gel or solid state as discussed in [82]. Finally, experimental approaches often investigate one protein at a time to decipher the physical nature of sub-compartments. However, some proteins could form a droplet while others crosslink chromatin in the same compartment. To challenge these ideas, there is a need to refine the criteria that discriminate these models by building a solid theoretical framework. Single molecule microscopy, coupled to the power of yeast genetics allowing rapid and well controlled perturbations, appears as a promising approach to challenge these models and to decipher the physical nature of sub-compartments in the nucleus.

\section{Acknowledgements:}

We would like to thank Aleksandra Walczak and Thierry Mora for fruitful discussions and comments about this work. We also thank Antoine Coulon, Susmita Sridhar and Myriam Ruault for helpful comments on the manuscript. Our team is financially supported by funding from the Labex DEEP (ANR-11-LABX-0044_DEEP and ANR-10-IDEX-0001-02 PSL), from the ANR DNA-Life and DICENs (ANR-15-CE12-0007; ANR-14-CE10-0021-04), and Fondation pour la Recherche Médicale grant DEP20151234398 and PIC3i (Interdisciplinary Program from the Institut Curie). We apologize to any colleagues whose work could not be cited due to space limitations.

\section{The authors declare no conflict of interest.}




\section{References:}

\section{Highlighted publications:}

Hocher et al., 2018:

Overexpressing the silencing factor Sir3 at varying levels, they found that Sir3 spreads into Extended Silent Domains (ESDs), eventually reaching saturation at subtelomeres. This is accompanied by increased telomere clustering but once maximal clustering is achieved, increasing Sir3 levels augment the signal in the nucleoplasm while Sir3 foci remain unchanged.

Miné-Hattab et al., 2017:

Using multi-scale tracking, they show that motion of damaged DNA is composed of several diffusion regimes that simultaneously drive DNA at each time scale. Following DNA damage, chromatin mobility is increased at large time scales but reduced at short time scales, consistent with a global chromatin stiffening, this effect being more pronounced at the damaged site.

Strom et al., 2017:

They show that Drosophila HP1a protein undergoes liquid-liquid demixing in vitro, and that in both Drosophila and mammalian cells heterochromatin domains exhibit dynamics that are characteristic of liquid-phase separation. They proposed that the formation of heterochromatin domains is mediated by phase separation and matures into a structure that exhibits liquid-like properties.

Boehning et al., , 2018:

Human and yeast carboxy-terminal domains (CTD) of RNA polymerase (Pol) II undergo cooperative liquid-phase separation in vitro, the shorter yeast CTDs forming less-stable droplets. In human cells, CTD length was shown to influence polII clustering and mobility in vivo. Finally, CTD phosphorylation regulates phase separation since CTD phosphorylation dissolves droplets.

Zhang et al., 2017:

They synthesized a 976.067 bp linear chromosome, synXII, based on the native chromosome XII in Saccharomyces cerevisiae. Cells deleted for the rDNA region and bearing single rDNA copies on a multicopy plasmid show dispersed "mini-nucleoli" (consistent with Oakes et al, 1998). In contrast, integration of a chimeric rDNA - in which the internal transcribed spacer (ITS) was replaced with those from the related yeast $S$. bayanus - at 3 different genomic locations, shows little effect on fitness and forms nucleolar structures indistinguishable from that of the wild type.

Hult et al., 2017 :

They modelled the rDNA by introducing crosslinking within and between chains representing chromatin interaction with SMC proteins, or with HMO1 involved in rDNA transcriptional regulation. They proposed that the nucleolus forms a gel-gel phase separation, where the nucleolus exhibits properties corresponding to a 'stiffer' gel compared to the rest of the genome, this phase separation being driven by the enrichment of protein-mediated crosslinking of chromatin within the rDNA. Consistent with their model, they further show that when the rDNA is split between two loci, a single nucleolus is formed. 
Schrank et al., 2018:

In Xenopus laevis cell free extracts and in human cells, they found that nuclear actin, WASP, and the actin-nucleating ARP2/3 complex are recruited to damaged chromatin undergoing homology-directed repair. Furthermore, nuclear actin polymerization promotes the migration of multiple double-strand breaks into discrete sub-nuclear clusters.

Swygert et al, 2018:

Reconstituted chromatin fibers with the Sir2/3/4 proteins were analyzed by sedimentation velocity, molecular modeling and atomic force microscopy to characterize the stoichiometry and conformation of SIR chromatin fibers. This data suggests a model in which one molecule of Sir2/4 binds primarily within each nucleosome linker and two molecules of Sir3 bind to each surface of the nucleosome, leading to a compact structure that effectively represses DNAtemplated reactions.

1. Hoze, N., et al., Spatial telomere organization and clustering in yeast Saccharomyces cerevisiae nucleus is generated by a random dynamics of aggregation-dissociation. Mol Biol Cell, 2013. 24(11): p. 1791-800, S1-10.

2. Taddei, A. and S.M. Gasser, Structure and function in the budding yeast nucleus. Genetics, 2012. 192(1): p. 107-29.

3. Rabl, C., On Cell Division. Morphologisches Jahrbuch. Morphologisches Jahrbuch, 1885. 10

p. 214-330 (in German).

4. Dekker, J., et al., Capturing chromosome conformation. Science, 2002. 295(5558): p. 1306-11.

5. Miné-Hattab, J. and R. Rothstein, Increased chromosome mobility facilitates homology search during recombination. Nat Cell Biol, 2012. 14(5): p. 510-7.

6. Heun, P., et al., Chromosome dynamics in the yeast interphase nucleus. Science, 2001. 294(5549): p. 2181-6.

7. Albert, B., et al., Systematic characterization of the conformation and dynamics of budding yeast chromosome XII. J Cell Biol, 2013. 202(2): p. 201-10.

8. Laporte, D., et al., Quiescent Saccharomyces cerevisiae forms telomere hyperclusters at the nuclear membrane vicinity through a multifaceted mechanism involving Esc1, the Sir complex, and chromatin condensation. Mol Biol Cell, 2016. 27(12): p. 1875-84.

9. Seeber, A., M.H. Hauer, and S.M. Gasser, Chromosome Dynamics in Response to DNA Damage. Annu Rev Genet, 2018.

10. Miné-Hattab, J. and R. Rothstein, DNA in motion during double-strand break repair. Trends Cell Biol, 2013. 23(11): p. 529-36.

11. Klein, H., ..., and A. Malkova, Guidelines for DNA recombination and repair studies: Cellular assays of DNA repair pathways. MicroBial Cell, 2018. in press.

12. Marshall, W.F., et al., Interphase chromosomes undergo constrained diffusional motion in living cells. Curr Biol, 1997. 7(12): p. 930-9.

13. Weber, S.C., A.J. Spakowitz, and J.A. Theriot, Bacterial chromosomal loci move subdiffusively through a viscoelastic cytoplasm. Phys Rev Lett, 2010. 104(23): p. 238102.

14. Hajjoul, H., et al., High-throughput chromatin motion tracking in living yeast reveals the flexibility of the fiber throughout the genome. Genome Res, 2013. 23(11): p. 1829-38.

15. Mine-Hattab, J., et al., Multi-scale tracking reveals scale-dependent chromatin dynamics after DNA damage. Mol Biol Cell, 2017.

16. Larson, D.R., et al., Real-time observation of transcription initiation and elongation on an endogenous yeast gene. Science, 2011. 332(6028): p. 475-8.

17. Arbona, J.M., et al., Inferring the physical properties of yeast chromatin through Bayesian analysis of whole nucleus simulations. Genome Biol, 2017. 18(1): p. 81.

18. Wong, H., J.M. Arbona, and C. Zimmer, How to build a yeast nucleus. Nucleus, 2013. 4(5): p. 361-6. 
19. Tjong, H., et al., Physical tethering and volume exclusion determine higher-order genome organization in budding yeast. Genome Res, 2012. 22(7): p. 1295-305.

20. Tokuda, N., T.P. Terada, and M. Sasai, Dynamical modeling of three-dimensional genome organization in interphase budding yeast. Biophys J, 2012. 102(2): p. 296-304.

21. Guidi, M., et al., Spatial reorganization of telomeres in long-lived quiescent cells. Genome Biol, 2015. 16: p. 206.

22. Dultz, E., et al., Global reorganization of budding yeast chromosome conformation in different physiological conditions. J Cell Biol, 2016. 212(3): p. 321-34.

23. Rutledge, M.T., et al., The yeast genome undergoes significant topological reorganization in quiescence. Nucleic Acids Res, 2015. 43(17): p. 8299-313.

24. Nagai, S., et al., Functional targeting of DNA damage to a nuclear pore-associated SUMOdependent ubiquitin ligase. Science, 2008. 322(5901): p. 597-602.

25. Ho, B., A. Baryshnikova, and G.W. Brown, Unification of Protein Abundance Datasets Yields a Quantitative Saccharomyces cerevisiae Proteome. Cell Syst, 2018. 6(2): p. 192-205 e3.

26. Mekhail, K., et al., Role for perinuclear chromosome tethering in maintenance of genome stability. Nature, 2008. 456(7222): p. 667-70.

27. Kobayashi, T., Regulation of ribosomal RNA gene copy number and its role in modulating genome integrity and evolutionary adaptability in yeast. Cell Mol Life Sci, 2011. 68(8): p. 1395-403.

28. Woolford, J.L., Jr. and S.J. Baserga, Ribosome biogenesis in the yeast Saccharomyces cerevisiae. Genetics, 2013. 195(3): p. 643-81.

29. Leger-Silvestre, I., et al., Functional compartmentalization of the nucleus in the budding yeast Saccharomyces cerevisiae. Chromosoma, 1999. 108(2): p. 103-13.

30. Shou, W., et al., Exit from mitosis is triggered by Tem1-dependent release of the protein phosphatase Cdc14 from nucleolar RENT complex. Cell, 1999. 97(2): p. 233-44.

31. Ouenzar, F., et al., Cell cycle-dependent spatial segregation of telomerase from sites of DNA damage. J Cell Biol, 2017. 216(8): p. 2355-2371.

32. Sydorskyy, Y., et al., A novel mechanism for SUMO system control: regulated Ulp1 nucleolar sequestration. Mol Cell Biol, 2010. 30(18): p. 4452-62.

33. Torres-Rosell, J., et al., The Smc5-Smc6 complex and SUMO modification of Rad52 regulates recombinational repair at the ribosomal gene locus. Nat Cell Biol, 2007. 9(8): p. 923-31.

34. Belagal, P., et al., Decoding the principles underlying the frequency of association with nucleoli for RNA polymerase III-transcribed genes in budding yeast. Mol Biol Cell, 2016. 27(20): p. 3164-3177.

35. Haeusler, R.A., et al., Clustering of yeast tRNA genes is mediated by specific association of condensin with tRNA gene transcription complexes. Genes Dev, 2008. 22(16): p. 2204-14.

36. Trumtel, S., et al., Assembly and functional organization of the nucleolus: ultrastructural analysis of Saccharomyces cerevisiae mutants. Mol Biol Cell, 2000. 11(6): p. 2175-89.

37. Misteli, T., Where the nucleus comes from. Trends Cell Biol, 2001. 11(4): p. 149.

38. Hernandez-Verdun, D., P. Roussel, and J. Gebrane-Younes, Emerging concepts of nucleolar assembly. J Cell Sci, 2002. 115(Pt 11): p. 2265-70.

39. Wang, R., et al., High resolution microscopy reveals the nuclear shape of budding yeast during cell cycle and in various biological states. J Cell Sci, 2016. 129(24): p. 4480-4495.

40. Sinclair, D.A. and L. Guarente, Extrachromosomal rDNA circles--a cause of aging in yeast. Cell, 1997. 91(7): p. 1033-42.

41. Mercy, G., et al., 3D organization of synthetic and scrambled chromosomes. Science, 2017. 355(6329).

42. Zhang, W., et al., Engineering the ribosomal DNA in a megabase synthetic chromosome. Science, 2017. 355(6329).

43. Palladino, F., et al., SIR3 and SIR4 proteins are required for the positioning and integrity of yeast telomeres. Cell, 1993. 75(3): p. 543-55.

44. Swygert, S., et al., SIR proteins create compact heterochromatin fibers. bioRxiv, 2018. 
45. Gartenberg, M.R. and J.S. Smith, The Nuts and Bolts of Transcriptionally Silent Chromatin in Saccharomyces cerevisiae. Genetics, 2016. 203(4): p. 1563-99.

46. Maillet, L., et al., Evidence for silencing compartments within the yeast nucleus: a role for telomere proximity and Sir protein concentration in silencer-mediated repression. Genes Dev, 1996. 10(14): p. 1796-811.

47. Marcand, S., et al., Silencing of genes at nontelomeric sites in yeast is controlled by sequestration of silencing factors at telomeres by Rap 1 protein. Genes Dev, 1996. 10(11): p. 1297-309.

48. Taddei, A., et al., The functional importance of telomere clustering: global changes in gene expression result from SIR factor dispersion. Genome Res, 2009. 19(4): p. 611-25.

49. Batte, A., et al., Recombination at subtelomeres is regulated by physical distance, doublestrand break resection and chromatin status. EMBO J, 2017. 36(17): p. 2609-2625.

50. Therizols, P., et al., Chromosome arm length and nuclear constraints determine the dynamic relationship of yeast subtelomeres. PNAS, 2011. 107(5): p. 2025-2030.

51. Schober, $\mathrm{H}$., et al., Controlled exchange of chromosomal arms reveals principles driving telomere interactions in yeast. Genome Res, 2008. 18(2): p. 261-71.

52. Duan, Z., et al., A three-dimensional model of the yeast genome. Nature, 2010. 465(7296): p. 363-7.

53. Meister, P. and A. Taddei, Building silent compartments at the nuclear periphery: a recurrent theme. Curr Opin Genet Dev, 2013. 23(2): p. 96-103.

54. Ruault, M., et al., Clustering heterochromatin: Sir3 promotes telomere clustering independently of silencing in yeast. J Cell Biol, 2011. 192(3): p. 417-31.

55. Smith, C.D., et al., Telomeric protein distributions and remodeling through the cell cycle in Saccharomyces cerevisiae. Mol Biol Cell, 2003. 14(2): p. 556-70.

56. Lisby, M., et al., Choreography of the DNA damage response: spatiotemporal relationships among checkpoint and repair proteins. Cell, 2004. 118(6): p. 699-713.

57. Bordelet, H. and K. Dubrana, Keep moving and stay in a good shape to find your homologous recombination partner. Curr Genet, 2018.

58. Haber, J.E., DNA Repair: The Search for Homology. Bioessays, 2018. 40(5): p. e1700229.

59. Short, J.M., et al., High-resolution structure of the presynaptic RAD51 filament on singlestranded DNA by electron cryo-microscopy. Nucleic Acids Res, 2016. 44(19): p. 9017-9030.

60. Shibata, T., et al., Homologous genetic recombination as an intrinsic dynamic property of a DNA structure induced by RecA/Rad51-family proteins: a possible advantage of DNA over RNA as genomic material. Proc Natl Acad Sci U S A, 2001. 98(15): p. 8425-32.

61. Miné, J., et al., Real-time measurements of the nucleation, growth and dissociation of single Rad51-DNA nucleoprotein filaments. Nucleic Acids Res, 2007. 35(21): p. 7171-87.

62. Haber, J.E., Mating-type genes and MAT switching in Saccharomyces cerevisiae. Genetics, 2012. 191(1): p. 33-64.

63. Dion, V., et al., Increased mobility of double-strand breaks requires Mec1, Rad9 and the homologous recombination machinery. Nat Cell Biol, 2012. 14(5): p. 502-9.

64. Jakob, B., et al., Live cell microscopy analysis of radiation-induced DNA double-strand break motion. Proc Natl Acad Sci U S A, 2009. 106(9): p. 3172-7.

65. Chiolo, l., et al., Double-strand breaks in heterochromatin move outside of a dynamic HP1a domain to complete recombinational repair. Cell, 2011. 144(5): p. 732-44.

66. Schrank, B.R., et al., Nuclear ARP2/3 drives DNA break clustering for homology-directed repair. Nature, 2018. 559(7712): p. 61-66. In Xenopus laevis cell free extracts and in human cells, they found that nuclear actin, WASP, and the actin-nucleating ARP2/3 complex are recruited to damaged chromatin undergoing homology-directed repair. Furthermore, nuclear actin polymerization promotes the migration of multiple double-strand breaks into discrete sub-nuclear clusters.

67. Lisby, M., U.H. Mortensen, and R. Rothstein, Colocalization of multiple DNA double-strand breaks at a single Rad52 repair centre. Nat Cell Biol, 2003. 5(6): p. 572-7. 
68. Bergeron-Sandoval, L.P., N. Safaee, and S.W. Michnick, Mechanisms and Consequences of Macromolecular Phase Separation. Cell, 2016. 165(5): p. 1067-1079.

69. Erdel, F. and K. Rippe, Formation of Chromatin Subcompartments by Phase Separation. Biophys J, 2018. 114(10): p. 2262-2270.

70. Woodruff, J.B., A.A. Hyman, and E. Boke, Organization and Function of Non-dynamic Biomolecular Condensates. Trends Biochem Sci, 2018. 43(2): p. 81-94.

71. Jost, D., C. Vaillant, and P. Meister, Coupling 1D modifications and 3D nuclear organization: data, models and function. Curr Opin Cell Biol, 2017. 44: p. 20-27.

72. Bugin, A., F. Brochard-Wyart, and P.-G. de Gennes, Effondrement d'une chaine flexible en mauvais solvant. C.R. Acad. Sci. Paris, 1996. 322(II-b).

73. Hult, C., et al., Enrichment of dynamic chromosomal crosslinks drive phase separation of the nucleolus. Nucleic Acids Res, 2017. 45(19): p. 11159-11173.

74. Hyman, A.A., C.A. Weber, and F. Julicher, Liquid-liquid phase separation in biology. Annu Rev Cell Dev Biol, 2014. 30: p. 39-58.

75. Haddad, N., D. Jost, and C. Vaillant, Perspectives: using polymer modeling to understand the formation and function of nuclear compartments. Chromosome Res, 2017. 25(1): p. 35-50.

76. Larson, A.G., et al., Liquid droplet formation by HP1alpha suggests a role for phase separation in heterochromatin. Nature, 2017. 547(7662): p. 236-240.

77. Strom, A.R., et al., Phase separation drives heterochromatin domain formation. Nature, 2017. 547(7662): p. 241-245.

78. Altmeyer, M., et al., Liquid demixing of intrinsically disordered proteins is seeded by poly(ADP-ribose). Nat Commun, 2015. 6: p. 8088.

79. Patel, A., et al., A Liquid-to-Solid Phase Transition of the ALS Protein FUS Accelerated by Disease Mutation. Cell, 2015. 162(5): p. 1066-77.

80. Boehning, M., et al., RNA polymerase II clustering through carboxy-terminal domain phase separation. Nat Struct Mol Biol, 2018. 25(9): p. 833-840.

81. Feric, M., et al., Coexisting Liquid Phases Underlie Nucleolar Subcompartments. Cell, 2016. 165(7): p. 1686-1697.

82. Banani, S.F., et al., Biomolecular condensates: organizers of cellular biochemistry. Nat Rev Mol Cell Biol, 2017. 18(5): p. 285-298.

83. Brangwynne, C.P., et al., Germline P granules are liquid droplets that localize by controlled dissolution/condensation. Science, 2009. 324(5935): p. 1729-32.

84. Hocher, A., et al., Expanding heterochromatin reveals discrete subtelomeric domains delimited by chromatin landscape transitions. accepted in 2018. 


\section{Figures:}

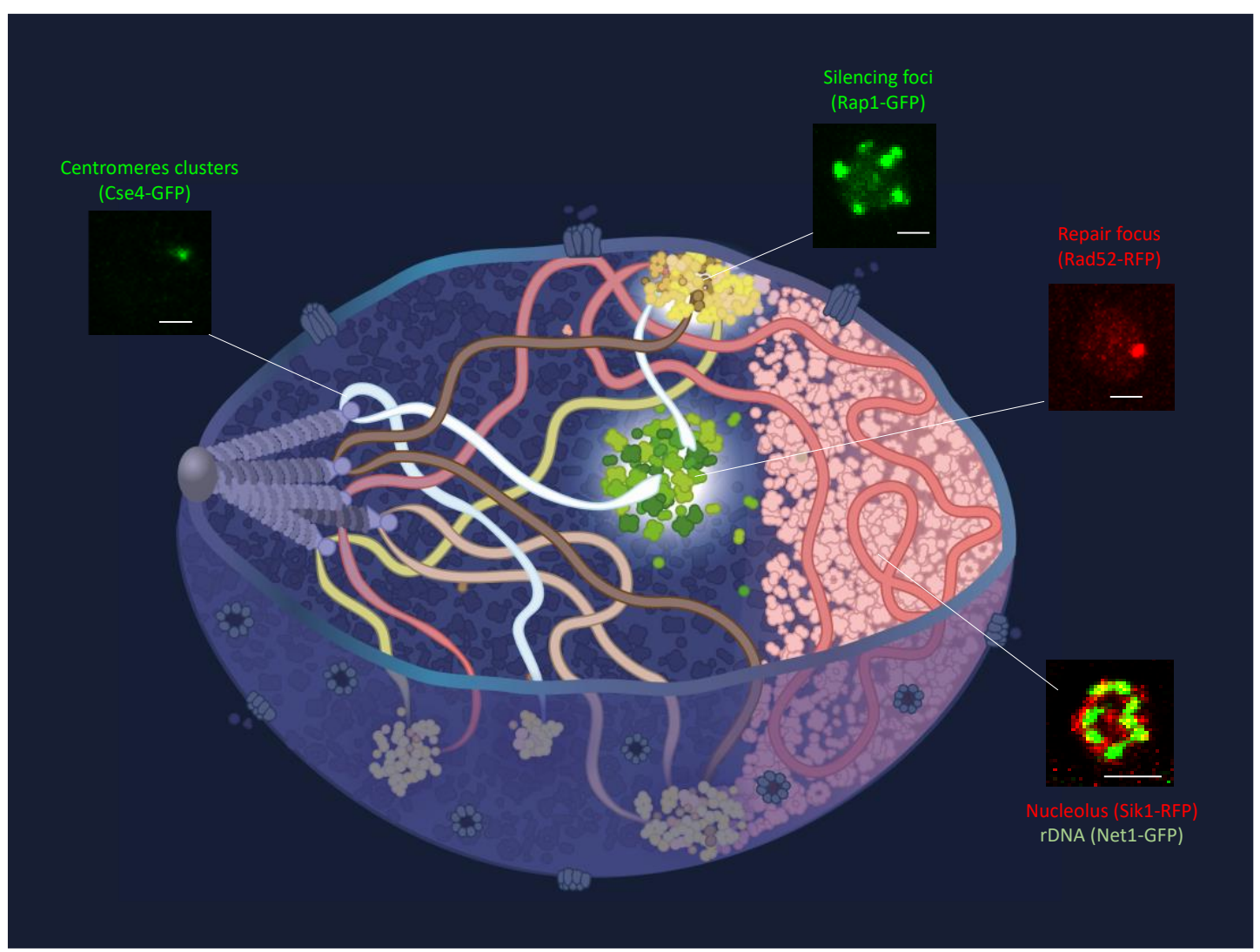

Figure 1: Illustration of a yeast nucleus in G1. Five of the 16 chromosomes are represented following the Rabl-like configuration. Chromosomes size ranges from $229 \mathrm{~kb}$ to $2.8 \mathrm{Mb}$. Microscopy images illustrates some of the membrane-less compartments. Rad52-RFP (MinéHattab et al, unpublished), Rap1-GFP and Net1-GFP/Sik1-RFP (courtesy of Myriam Ruault). Scale-bars are $1 \mu \mathrm{m}$. (Illustrator: Olga Markova).
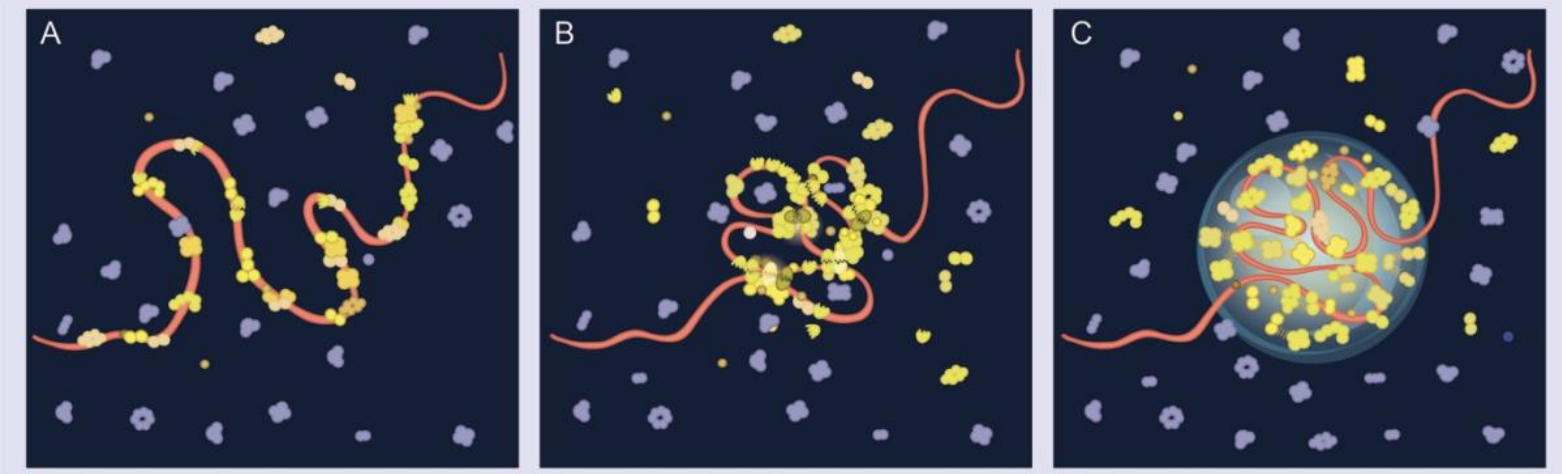

Figure 2: Illustration of the 3 models of membrane-less compartments presented in the text. A) The "binding model": sub-compartments are formed by proteins binding to available chromatin sites and no phase separation emerges. B) The "bridging model": proteins are able to bridge distant chromatin loci closer and a polymer-phase separation emerges. C) The "droplet model: proteins inside a sub-compartment create a micro-environment with liquidlike properties. These 3 models are not exclusive of one another and binding events are often proposed as the nucleation core both for the bridging and the droplet models. (Illustrator: Olga Markova). 